\title{
ESTUDO TERMODINÂMICO E CINÉTICO DA DESSULFURAÇÃO DE FERRO GUSA UTILIZANDO TERMODINAMICA COMPUTACIONAL*
}

\section{Resumo}

Heitor Cristo Clem de Oliveira ${ }^{1}$ Silas Gambarine Soares ${ }^{2}$ Anna Paula Littig Berger ${ }^{3}$ Rodrigo Pezzin de Oliveira ${ }^{4}$ Ludimila Melo Vieira ${ }^{5}$ José Roberto de Oliveira ${ }^{6}$

O objetivo deste trabalho é estudar a reação de dessulfuração, envolvidas no processo de pré-tratamento de ferro-gusa. A princípio, as misturas utilizadas foram caracterizadas e estudadas através do software de termodinâmica computacional (FactSage 7.1). Em seguida, os resultados obtidos através do estudo termodinâmico computacional foram comparados com aqueles obtidos mediante os testes experimentais a $1400^{\circ} \mathrm{C}$. As misturas dessulfurantes tiveram como base a $\mathrm{CaO}$ com três tipos de fundentes, fluorita, sodalita e barrilha, além da utilização de alumínio como elemento desoxidante. Os testes experimentais permitiram identificar que a adição de $0,1 \%$ em massa de alumínio no gusa, eleva a taxa de remoção de enxofre principalmente no instantes iniciais do processo. Com a utilização da termodinâmica computacional pode-se mostrar que a reação de dessulfuração no ferro-gusa é exotérmica para os teores de silício encontrados no processo. Além disso, a termodinâmica computacional também contribui, em alguns casos, para a análise de compostos sólidos que podem se formar na escória e limitar as reações de dessulfuração.

Palavras-chave:Dessulfuração; Termodinâmica Computacional; Ferro-gusa.

\section{THERMODYNAMIC AND KINETICAL STUDY OF HOT METAL DES USING THERMODYNAMIC COMPUTATIONAL}

\section{Abstract}

The aim of this project is to study the desulphurization reaction involved in the pretreatment of the hot metal. At first, the mixtures used were characterized and studied through the thermodynamics computational software (FactSage 7.1). The results gived by the software were compared with those obtained by the experimental tests at 1400 ${ }^{\circ} \mathrm{C}$. The desulphurising mixtures were based on $\mathrm{CaO}$ with three types of fluxes, fluorite, sodalite and Sodium Carbonate, besides the use of aluminum as a deoxidizing element. The experimental tests allowed identifying that the addition of $0.1 \%$ by mass of aluminum in the hot metal, raises the rate of sulfur removal mainly at the initial instants of the process. With the use of computational thermodynamics showed that the desulfurization reaction in hot metal is exothermic for the silicon contents found in the process. In addition, computational thermodynamics also contribute, in some cases, to the analysis of solid compounds that may form in the slag and limit the desulphurization reactions.

Keywords: Desulphurisation; Computational Thermodynamics; Hot Metal.

\footnotetext{
Mestre em Eng. Metalúrgica e de Materiais, Instituto Federal do Espírito Santo, Vitória, ES, Brasil. Mestre em Engenharia Metalúrgica e de Materiais, IFES, Vitória, ES, Brasil.

Eng. Metalurgista e Mestranda em Engenharia Metalúrgica e de Materiais, IFES, Vitória, ES, Brasil. Eng. Metalurgista e Mestrando em Eng. Metalúrgica e de Materiais, IFES, Vitória, ES, Brasil.

Eng. Metalurgista e Mestranda em Eng. Metalúrgica e de Materiais, IFES, Vitória, ES, Brasil.

Prof. Dr. do IFES, cursos de graduação e pós-graduação em Eng. Metalúrgica e de Materiais.
} 


\section{INTRODUÇÃO}

A dessulfuração de ferro-gusa normalmente é representada pela (Equação 1). [1]

$$
\begin{array}{r}
\mathrm{CaO}_{(\mathrm{s})}+\underline{\mathrm{S}}=\mathrm{CaS}_{(\mathrm{s})}+\underline{\mathrm{O}} \\
\Delta \mathrm{G}^{\circ}{ }_{1=1}=15358-38,66 \cdot \mathrm{T}(\mathrm{J} / \mathrm{mol})
\end{array}
$$

Porém, para os teores de silício contido no ferro-gusa, o oxigênio liberado pela equação 1 pode reagir com estes elementos, conforme a (Equação 3). [2]

$$
\begin{array}{r}
2 \mathrm{CaO}_{(\mathrm{s})}+1 / 2 \underline{\mathrm{Si}}+\underline{\mathrm{S}}=1 / 22 \mathrm{CaO}^{-\mathrm{SiO}_{2}(\mathrm{~s})}+\mathrm{CaS}_{(\mathrm{s})} \\
\Delta \mathrm{G}_{3}{ }^{\circ}=-246222+78 \mathrm{~T}
\end{array}
$$

Nota-se que, para as temperaturas de trabalho do ferro-gusa, a (Equação 1) é endotérmica $\left({ }^{1300^{\circ} \mathrm{C}} \Delta \mathrm{G}^{\circ}{ }_{1}=54545.82 \mathrm{~J} / \mathrm{mol}\right.$ e ${ }^{1450^{\circ} \mathrm{C}} \Delta \mathrm{G}^{\circ}{ }_{1}=48746.82 \mathrm{~J} / \mathrm{mol}$ ) e a (Equação

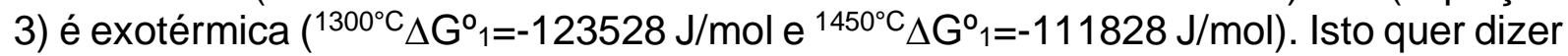
que, na presença de silício, o aumento da temperatura prejudica a remoção de enxofre.

Niedringhaus e Fruehan [3] apresentaram a (Equação 5) quando se tem a presença de alumínio com a cal para dessulfuração em gusa.

$$
\begin{array}{r}
3 \mathrm{CaO}_{(\mathrm{s})}+3 \underline{\mathrm{S}}+\underline{\mathrm{Al}}=\mathrm{Al}_{2} \mathrm{O}_{3}(\mathrm{~s})+3 \mathrm{CaS}_{(\mathrm{s})} \\
\Delta \mathrm{G}_{5}{ }^{\circ}=-319,343+111,3 T(\mathrm{~J} / \mathrm{mol})
\end{array}
$$

Niedringhaus e Fruehan [3] afirmam que a melhoria na eficiência de dessulfuração quando se adiciona Alumínio, é devido à diminuição das fases solidas que se forma em torno da partícula de cal e não devido à diminuição no teor de oxigênio do banho. Choi e et al [4] propuseram o modelo representado pela (Equação 7), para avaliar a cinética da reação de dessulfuração de gusa.

$$
-\frac{d[\% S]}{d t}=\left(k_{m}+k_{s} \cdot L_{s}+k_{f}\right) \cdot\left(\frac{A}{V_{m}}\right) \cdot\left\{[\% S]-\left[\% S_{e q}\right]\right\}
$$

Onde:

k: Coeficiente de transferência de massa do enxofre $(\mathrm{m} / \mathrm{s}) ; \mathrm{m}, \mathrm{s}$, f: Denotam a fase metal, a fase escória e a interface metal-escória, respectivamente; A: Área Interfacial $\left(m^{2}\right)$ e $V_{m}=$ Volume de metal $\left(m^{3}\right)$.

A (Equação 7) pode ser simplificada, uma vez que o termo $\left(k_{m}+k_{s} \cdot L_{s}+k_{f}\right)$ pode ser substituído pelo coeficiente de transferência de massa global $\left(k^{\prime}\right)$. Assim, a (Equação 7) se reduz a (Equação 8).

$$
-\frac{d[\% S]}{d t}=k^{\prime} \cdot\left(\frac{A}{V_{m}}\right) \cdot\left\{[\% S]-\left[\% S_{e q}\right]\right\}
$$

Porém devido à dificuldade de determinar alguns parâmetros, a (Equação 8) pode ser reduzida para a (Equação 9):

$$
\frac{d[\% S]}{d t}=-\mathrm{K}\left([\% S]-[\% S]_{e}\right)
$$


Onde $K=k^{\prime}\left(\frac{A}{V_{m}}\right)$ e é a constante de velocidade $\left(\min ^{-1}\right.$ ou $\left.\mathrm{s}^{-1}\right)$. K é função do transporte de massa do enxofre no metal e na escória. $O$ valor de $k$ pode ser determinado pela (Equação 10).

$$
\ln \left(\frac{\left[\% S_{0}\right]-\left[\% S_{e q}\right]}{\left[\% S_{t}\right]-\left[\% S_{e q}\right]}\right)=k . t
$$

De acordo com Furehan, esta equação é válida quando não há fase solida formada e torno da partícula de cal.

Lindström et al [5] descrevem que, no processo de dessulfuração, é comum a formação de uma camada de ( $\left.\mathrm{CaS}, 2 \mathrm{CaO} . \mathrm{SiO} 2,2 \mathrm{CaO} . \mathrm{Al}_{2} \mathrm{O}_{3}\right)$ em volta das partículas de $\mathrm{CaO}$, à medida que o processo dessulfuração evolui. Além disso, devido ao estado de agitação, pode-se admitir a inexistência da camada limite no gusa, ou caso exista, sua espessura pode ser considerada insignificante. Sendo assim, conforme relatado por Lindström et al [6], a espessura da camada de $\mathrm{CaS}, 2 \mathrm{CaO} . \mathrm{SiO} 2,2 \mathrm{CaO} . \mathrm{Al}_{2} \mathrm{O}_{3}$ sobre a partícula de $\mathrm{CaO}$, varia em função do tempo, seguindo a (Equação 11):

Onde:

$$
\delta=c \sqrt{t}
$$

c: constante de proporcionalidade; t: tempo; $\delta$ : espessura da camada (CaS, $\left.2 \mathrm{CaO} . \mathrm{SiO}_{2}, 2 \mathrm{CaO} \cdot \mathrm{Al}_{2} \mathrm{O}_{3}\right)$.

Desse modo a dessulfuração é dependente de um processo de difusão, e assim sendo, os fatores que afetam a difusão, afetam a cinética da dessulfuração como por exemplo as fases sólidas formadas em volta da partícula de cal.

Isto então sugere que a equação cinética tem que levar em conta a espessura da fase solida que muda com o tempo. Assim, a termodinâmica computacional pode determinar dados que esclarecem melhor estes mecanismos.

\section{MATERIAIS E MÉTODOS}

\subsection{Testes experimentais}

Para a realização deste trabalho, inicialmente foi definida a composição química das misturas dessulfurantes, de modo a se manter constante a massa de $\mathrm{CaO}$ em $10 \mathrm{~kg} /$ ton de gusa e $5 \%$ de fundente. As composições químicas das misturas usadas são mostradas na Tabela 1:

\begin{tabular}{|c|c|c|c|c|c|c|c|c|c|}
\hline \multirow{2}{*}{ Misturas } & \multicolumn{8}{|c|}{ Composição Química (\%) } & \multirow{2}{*}{$\begin{array}{c}\text { Massa } \\
\text { total } \\
\text { (g) }\end{array}$} \\
\hline & $\mathrm{CaO}$ & $\mathrm{Na}_{2} \mathrm{O}$ & $\mathrm{CaF}_{2}$ & $\mathrm{Al}_{2} \mathrm{O}_{3}$ & $\mathrm{SiO}_{2}$ & $\mathrm{~K}_{2} \mathrm{O}$ & $\mathrm{MgO}$ & $\mathrm{Fe}_{2} \mathrm{O}_{3}$ & \\
\hline Alum+ $5 \% \mathrm{CaF}_{2}$ & 92,51 & 0 & 4,81 & 0,37 & 1,76 & 0 & 0,56 & 0 & 10,81 \\
\hline $5 \% \mathrm{CaF}_{2}$ & 92,51 & 0 & 4,81 & 0,37 & 1,76 & 0 & 0,56 & 0 & 10,81 \\
\hline 5\% Barrilha & 92 & 5,34 & 0 & 0,37 & 1,75 & 0 & 0,55 & 0 & 10,81 \\
\hline $5 \%$ Sodalita & 93,2 & 0,65 & 0 & 1,49 & 4,1 & 0,28 & 0,09 & 0,19 & 10,81 \\
\hline
\end{tabular}

Tabela 1 Composição química em porcentagem massa das misturas dessulfurantes propostas. 
Desse modo, é possível se comparar a influência e determinar qual é o melhor fundente na dessulfuração de gusa, além de averiguar o efeito da adição de alumínio no ferro-gusa, mantendo sempre a mesma proporção de $\mathrm{CaO}$. Já a composição química do gusa utilizado pode ser visto na Tabela 2:

Tabela 2. Composição química inicial do ferro-gusa

\begin{tabular}{ccccccc}
\hline \multicolumn{7}{c}{ Composição química (\%) } \\
\hline $\mathbf{F e}$ & $\mathbf{C}$ & $\mathbf{S i}$ & $\mathbf{M n}$ & $\mathbf{P}$ & $\mathbf{S}$ & $\mathbf{0}$ \\
\hline 94,739 & 4,6 & $0 \sim 0,2$ & 0,3 & 0,11 & 0,05 & 0,001 \\
\hline
\end{tabular}

Para realização dos experimentos foram utilizados cadinhos de grafite de alta pureza para fusão de $1 \mathrm{~kg}$ de gusa por experimento. Os testes foram realizados em atmosfera de argônio comercial, a uma vazão de aproximadamente $5 \mathrm{Nl} / \mathrm{mim}$. A injeção de argônio foi iniciada junto com o início do aquecimento e permaneceu durante todo o tempo dos experimentos. Uma vez atingida a temperatura de trabalho, $1400^{\circ} \mathrm{C}$, foi verificada a completa fusão do gusa por meio de uma entrada secundária, sendo retirada uma amostra inicial através de um amostrados de quartzo a vácuo (para análise de enxofre inicial) e em seguida, feitas as adições dos materiais dessulfurantes. Para auxiliar nas adições, foi utilizado um tubo de aço inoxidável visando direcionar o material para dentro do cadinho. A Figura 1 mostra um esquema da retirada de amostras durante os testes experimentais.

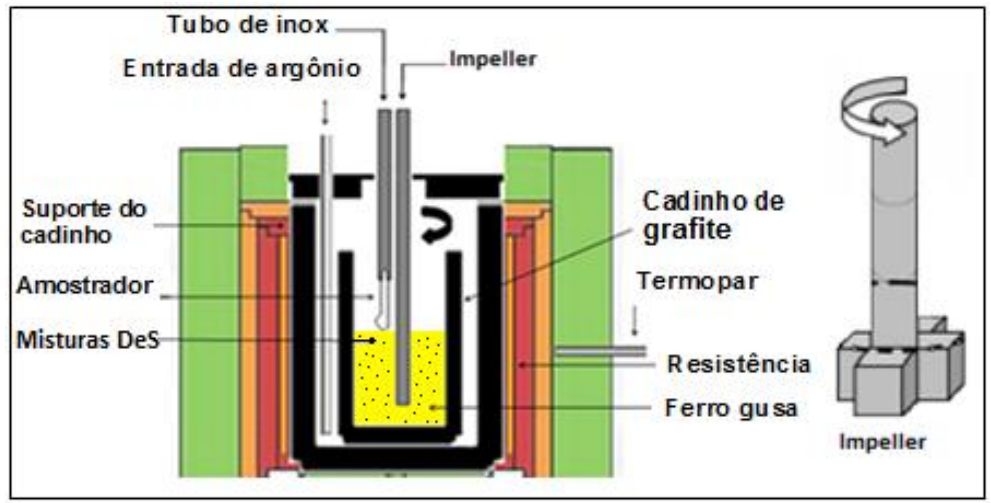

Figura 1. Diagrama esquemático dos experimentos realizados.

Após a adição das misturas dessulfurantes, foi ligado o agitador mecânico com a rotação do impeller a 400rpm. O tempo de reação passou a ser contado imediatamente após o carregamento. Foram retiradas amostras antes das adições e nos tempos de 5, 10, 15, 20 e 30 minutos após as adições. As retiradas de amostras também foram feitas pela entrada localizada no centro da tampa do forno, por meio de amostradores à vácuo. Cada amostra pesava aproximadamente 10 gramas.

\section{2. - Cálculos termodinâmicos computacionais;}

Os cálculos termodinâmicos computacionais foram realizados com o software FactSage 7.1, onde os bancos de dados utilizados para a avaliação das misturas e escórias foram o Factps, FToxid e FTMisc. Entretanto, em cada banco de dados escolhido existe um sub-banco de dados que atua de forma a selecionar de maneira mais precisa os compostos presentes na mistura.

O sub-banco de dados Pure Solids presente no banco de dados Factps é utilizado para a avaliação dos compostos sólidos formados na mistura. Já o sub-banco de 
dados SlagA e SlagH (escórias com fluorita) são utilizados para a avaliação dos compostos formados na fase líquida das misturas. $O$ banco de dados utilizados para a avaliação das condições de equilíbrio termodinâmico da reação de dessulfuração foi o FTmisc. Vale ressaltar que o sub-banco de dados selecionado foi o LIQUID, uma vez que a $1400^{\circ} \mathrm{C}$ o ferro-gusa está totalmente líquido.

\section{3. - Verificar para quais teores de Silício no gusa a Dessulfuração é exotérmica;}

Foi variado a porcentagem de silício inicial de $0 \%$ até $0,2 \%$ de modo a se verificar até qual teor desse elemento, a reação de dessulfuração é exotérmica. Para isto, foram traçadas curvas \% $\mathrm{S}_{\text {eq }} \times$ Temperatura para diferentes teores de $\mathrm{Si}$. As simulações foram realizadas utilizando uma composição química de ferro-gusa de aciaria mostrado na Tabela 1.

Como o objetivo das simulações era avaliar a influência do silício na dessulfuração de ferro-gusa, foi desconsiderado os valores de fosforo presentes no metal, uma vez que seu coeficiente de interação com o enxofre é elevado $\left(e_{S}^{P}=0,29\right)$, alterando a atividade do enxofre e gerando interferência nos resultados.

Como fonte de agente dessulfurante foi utilizado apenas $\mathrm{CaO}$ puro, na proporção de $10 \mathrm{~kg}$ de $\mathrm{CaO}$ para cada tonelada de ferro gusa.

\section{4. - Verificar se a presença de Al nas misturas dessulfurantes diminuem as fases solidas e o teor de enxofre de equilíbrio;}

Para avaliar a influência da adição de alumínio na dessulfuração de gusa, foram feitas simulações para determinar as fases presentes em uma escória a base de Cal (10kg/ton de gusa), variando sua concentração em $0,01 \%$ de 0 até $0,3 \%$. A composição do gusa utilizado foi de $\mathrm{C}=4,5 \%, \mathrm{~S}=0,12 \%, \mathrm{Si}=0,5 \%$ e $\mathrm{Mn}=0,4 \%$, de forma a simular computacionalmente os testes realizados por Niedringhaus e Fruehan [3].

\subsection{Determinação da composição química das escórias finais através do balanço de massa}

Como não foi possível a análise química da escória formada após a realização dos experimentos, devido à pequena quantidade de mistura dessulfurante adicionada (cerca de 10 gramas), foi feito um balanço de massa para determinar a composição química da escória final. Para isto, considerou-se eu todo o enxofre removido do banho metálico reagiu com a cal formando CaS e o oxigênio liberado da partícula de $\mathrm{CaO}$ oxidando o silício dissolvido no gusa, formando o $\mathrm{SiO}_{2}$.

Com isso, a composição química da escória final se dá pela variação de $\mathrm{CaO}$ e $\mathrm{SiO}_{2}$, ou seja, redução da quantidade de cal e aumento do teor de sílica, adicionando o CaS formado. Os valores para os outros componentes foram mantidos.

\section{6 - Comprovar que a espessura da fase sólida dever ser levada em conta na equação de DeS. .}

As análises cinéticas foram feitas em cima dos testes experimentais das misturas mostradas na Tabela 1. Assim pode-se traçar as curvas $-\ln \left(\frac{\left[\% S_{0}\right]-\left[\% S_{e q}\right]}{\left[\% S_{t}\right]-\left[\% S_{e q}\right]}\right) \times \mathrm{t} \mathrm{e}$ verificar suas correlações, de modo a identificar que, se para as escórias que formam 
fases sólidas, existe uma limitação cinética devido ao crescimento desses compostos sólidos sob a partícula de $\mathrm{CaO}$.

\section{RESULTADOS E DISCUSSÃO}

\subsection{Resultados dos Testes Experimentais}

A eficiência de dessulfuração (\%ฤ) foi calculado através da seguinte (Equação 12):

$$
\eta(\%)=\frac{\left[\% S_{i}\right]-\left\lfloor \% S_{f}\right\rfloor}{\left[\% S_{i}\right]} \times 100
$$

Onde $\left[\% S_{f}\right]$ é a concentração de enxofre final, e [\% $\left.\mathrm{S}_{i}\right]$ é a concentração inicial de enxofre no ferro-gusa.

A Figura 2 mostra a variação da concentração do enxofre com o tempo e a Tabela 3 mostra os teores da \%S inicial, final, equilíbrio e a eficiência de dessulfuração (n).

Tabela 3. Porcentagem de \% $S_{\text {incial }}, \% S_{\text {final, }} \% S_{\text {eq }}$ e $\eta$.

\begin{tabular}{|c|c|c|c|c|}
\hline Misturas & $\% S_{\text {inicial }}$ & $\% \mathrm{~S}_{\text {final }}$ & $\% \mathrm{~S}_{\text {eq }}$ & n (\%) \\
\hline $\begin{array}{c}\text { Alum+ 5\% } \\
\mathrm{CaF}_{2}\end{array}$ & 0,0392 & 0,0015 & 1,50E-05 & 96,17 \\
\hline $5 \% \mathrm{CaF}_{2}$ & 0,0418 & 0,0017 & 4,71E-05 & 95,33 \\
\hline 5\% Barrilha & 0,0427 & 0,0133 & 5,45E-05 & 68,85 \\
\hline $5 \%$ Sodalita & 0,0384 & 0,0208 & 5,36E-05 & 45,83 \\
\hline
\end{tabular}

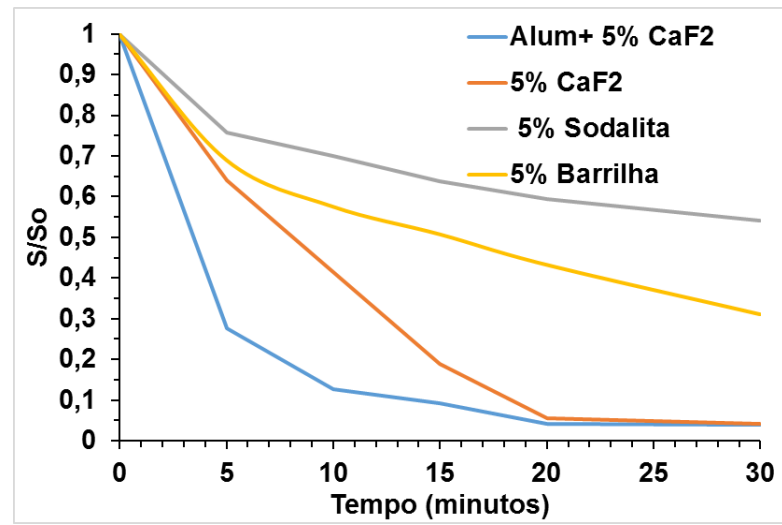

Figura 2. Variação da concentração de enxofre com o tempo

Pela Tabela 3 nota-se que a capacidade termodinâmica das escórias de remover enxofre é praticamente a mesma, pois os valores de \%Seq, foram praticamente iguais. Também pose-se observar que em nenhum experimento foi alcançado o equilíbrio. A adição de alumínio elevou a remoção de enxofre, principalmente nos instantes iniciais, uma vez que este elemento reduz o potencial de oxigênio do banho metálico. As misturas com Fluorita apresentaram uma maior eficiência em comparação com a de Barrilha e Sodalita. Esse resultado está de acordo com o encontrado por Grillo [7] e Oliveira et al [8], sendo que esses autores afirmam que a presença da sílica e alumina na sodalita promove a formação de compostos sólidos ao redor da partícula de cal, prejudicando a remoção de enxofre do banho metálico. 


\subsection{Resultados obtidos através da Termodinâmica Computacional}

\subsubsection{Influência do Silício nos valores de enxofre de equilíbrio no ferro-gusa}

Para avaliar a influência do silício na reação de dessulfuração, foram realizadas simulações termodinâmicas pelo software FactSage 7.1 variando seus teores de 0 a $0,2 \%$, que pode ser observado na Figura 3.
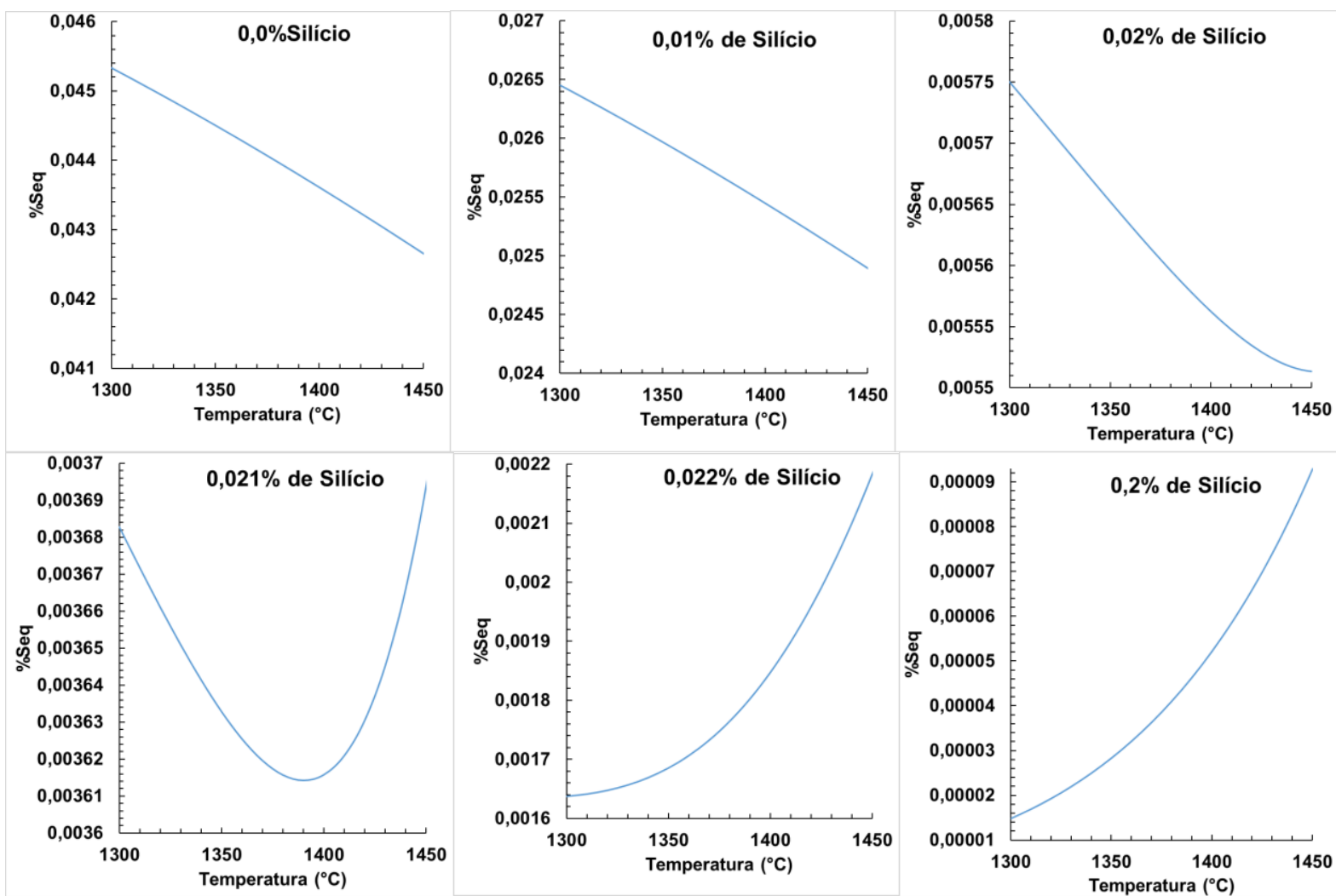

Figura 3. Variação da concentração de enxofre com o tempo

Analisando da Figura 3, pode-se observar que a reação de dessulfuração é endotérmica para valores de silício até $0,02 \%$, seguindo a (Equação 1 ), o que significa que o aumento de temperatura favorece a reação, diminuindo o enxofre de equilíbrio. Quando se eleva em $0,001 \%$ de Si na sua concentração, ocorre uma mudança no tipo de reação $\left(\operatorname{aos} 1390^{\circ} \mathrm{C}\right)$ tornando-a exotérmica, sendo representada pela (Equação $3)$.

A partir de $0,022 \%$ de silício, a reação já passa a ser totalmente exotérmica. Entretanto, mesmo o aumento da temperatura elevando os valores de enxofre de equilíbrio e desfavorecendo a reação, a cinética da reação é favorecida, uma vez que os compostos sólidos tendem a se tornarem líquidos (devido ao aumento da temperatura) e se juntar a escória líquida.

\subsubsection{Influência do Alumínio nos valores de enxofre de equilíbrio no ferro-gusa}

As simulações termodinâmicas foram realizadas variando os teores de Al de 0 a $0,3 \%$, em duas faixas de temperaturas, que pode ser observado na Figura 4 e Tabela 4. 
Tabela 4. Variação da \% $S_{\text {eq }}$ pela adição de Al.

\begin{tabular}{crr}
\hline $\begin{array}{c}\text { \%Al no } \\
\text { gusa }\end{array}$ & $\begin{array}{r}\text { \%S }_{\text {eq }} \mathbf{a} \\
\mathbf{1 3 0 0}^{\circ} \mathbf{C}\end{array}$ & $\begin{array}{c}\% \mathbf{S}_{\text {eq }} \mathbf{a} \\
\mathbf{1 4 5 0}^{\circ} \mathbf{C}\end{array}$ \\
\hline 0 & $1,74 \mathrm{E}-05$ & $5,72 \mathrm{E}-05$ \\
0,05 & $1,70 \mathrm{E}-05$ & $5,62 \mathrm{E}-05$ \\
0,1 & $1,53 \mathrm{E}-05$ & $4,87 \mathrm{E}-05$ \\
0,15 & $8,22 \mathrm{E}-06$ & $3,37 \mathrm{E}-05$ \\
0,2 & $5,99 \mathrm{E}-06$ & $2,46 \mathrm{E}-05$ \\
0,25 & $4,83 \mathrm{E}-06$ & $1,98 \mathrm{E}-05$ \\
0,3 & $4,10 \mathrm{E}-06$ & $1,68 \mathrm{E}-05$ \\
\hline
\end{tabular}

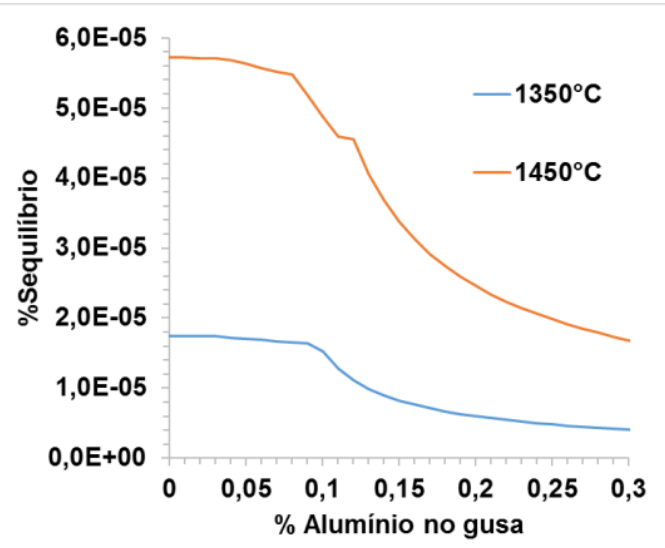

Figura 4. Variação da \% eq pela adição de Al.

Pela Tabela 4, nota-se que o aumento da concentração de Alumínio gera uma diminuição dos teores de enxofre de equilíbrio. Esse fato ocorre, pois, o Alumínio diminui o potencial de oxigênio do banho metálico. Já a Tabela 5 e Figura 5 mostram os resultados das simulações da variação da fase líquida pela adição de alumínio ao banho metálico.

Tabela 5. Variação da \%Líquido pela adição de Alumínio.

\begin{tabular}{ccc}
\hline $\begin{array}{c}\text { \%Al no } \\
\text { gusa }\end{array}$ & $\begin{array}{c}\text { \%Líquido } \\
\text { a } \mathbf{1 3 5 0} \mathbf{C}\end{array}$ & $\begin{array}{c}\text { \%Líquido } \\
\mathbf{a} \mathbf{1 4 5 0} \mathbf{C}\end{array}$ \\
\hline 0 & 0 & 0 \\
0,05 & 0 & 9,10 \\
0,1 & 0 & 27,29 \\
0,15 & 0 & 0 \\
0,2 & 0 & 0 \\
0,25 & 0 & 0 \\
0,3 & 0 & 0 \\
\hline
\end{tabular}

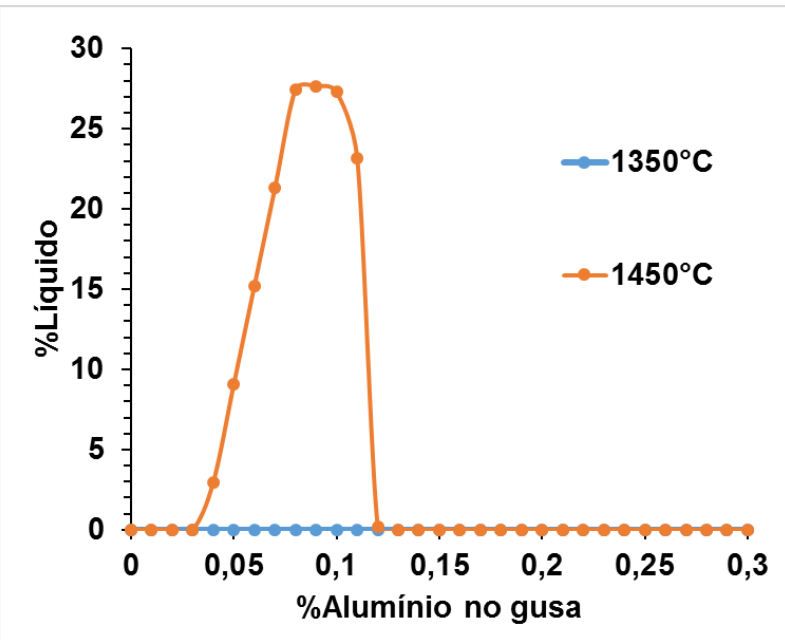

Figura 5. Variação da \%Líquido pela adição de Al.

Analisando a Tabela 5 e Figura 5 observa-se que na temperatura de $1450^{\circ} \mathrm{C}$ houve a formação de escória líquida para faixa de \% de alumínio de $0,04 \%$ a $0,12 \%$. Nos testes realizados por Niedringhaus e Fruehan [3], houve um grande aumento na taxa de dessulfuração quando se elevou a temperatura de $1350^{\circ} \mathrm{C}$ para $1450^{\circ} \mathrm{C}$, com $0,07 \%$ de Alumínio. Eles afirmaram que a $1450^{\circ} \mathrm{C}$ o Al reagiu com o Oxigênio e formou $\mathrm{Al}_{2} \mathrm{O}_{3}$ na escória líquida, o que não aconteceu a $1350^{\circ} \mathrm{C}$. Assim, eles concluem que o alumínio diminui a atividade do oxigênio e aumenta a força motriz na dessulfuração, mas isso deve ser secundário à formação do produto da reação em fase líquida, uma vez que para temperaturas na temperatura de $1350^{\circ}$ mesmo apresentando um menor enxofre de equilíbrio, ocorrerá uma maior formação de fazes sólidas que tendem a reduzir a cinética do processo. 


\subsubsection{Análise dos resultados experimentais}

Foram então realizadas simulações das condições de equilíbrio para as misturas dessulfurantes a $1400^{\circ} \mathrm{C}$, sem a presença do ferro-gusa, a fim de identificar a quantidade de líquido e sólido formado nessas escórias, para a temperatura de trabalho. A Tabela 6 mostra os resultados obtidos para o equilibro apenas das escórias a $1400^{\circ} \mathrm{C}$, obtidas pelo FactSage:

Tabela 6. Fases formadas nas misturas dessulfurantes utilizando o FactSage a $1400^{\circ} \mathrm{C}$.

\begin{tabular}{ccccccc}
\hline \multirow{2}{*}{ Misturas } & Sólidos & \multicolumn{3}{c}{$\%$ de Fases Sólidas } & Líquidos & \multirow{2}{*}{$(\%)$} \\
\cline { 3 - 5 } & & $\mathbf{C a O}$ & $\mathbf{M g O}$ & $\mathrm{Ca}_{3} \mathrm{SiO}_{5}$ & $(\%)$ & \multirow{2}{*}{ (\%) } \\
\hline Alum+ $5 \% \mathrm{CaF}_{2}$ & 89,01 & 88,09 & 0,54 & 0 & 10,99 & 96,17 \\
$5 \% \mathrm{CaF}_{2}$ & 89,01 & 88,09 & 0,54 & 0 & 10,99 & 95,33 \\
$5 \%$ Barrilha & 95,72 & 93,42 & 0,47 & 0 & 4,28 & 68,85 \\
$5 \%$ Sodalita & 90,23 & 83,89 & 0 & 2,17 & 9,77 & 45,83 \\
\hline
\end{tabular}

$\mathrm{Na}$ análise dos dados da Tabela 6 , nota-se que as escórias com o uso de $5 \%$ de fluorita, apresentaram maiores porcentagens de fase líquida a $1400^{\circ} \mathrm{C}$, quando comparadas com as escórias com barrilha. Verificou-se que o aumento da concentração de $\mathrm{SiO} 2$, favoreceu a formação dos compostos sólidos de silicato tricálcio (3CaO.SiO2). De acordo com Niedringhaus e Fruehan [3], que estudaram a influência da camada sólida no processo de dessulfuração de ferro-gusa, o aumento da quantidade dos compostos (3CaO.SiO2, 3CaO.Al2O3, 2CaO.SiO2) na escória, prejudicam a dessulfuração. De acordo com esses estudos, uma camada desses compostos se forma ao redor das partículas de $\mathrm{CaO}$, prejudicando a difusão do enxofre e aumentando a temperatura de fusão da partícula.

Grillo [7] verificou que a fase líquida tem papel secundário na dessulfuração do ferrogusa. Visto que, a fração de fase líquida é menor em relação a fase sólida. Neste caso, a remoção do enxofre no metal é realizada pela partícula sólida do $\mathrm{CaO}$, que através da agitação é levada até o seio do metal, reagindo com o enxofre e formando CaS.

Grillo [7] afirma que a fase líquida tem o papel de dissolver o CaS e o silicato tricálcio formado, evitando que estas fases se formem em torno da partícula de $\mathrm{CaO}$ e prejudiquem o transporte de massa do enxofre até a partícula de $\mathrm{CaO}$. Este comportamento da fase líquida fica evidente quando é realizado um balanço de massa para conhecer a composição das escórias finais e, posteriormente, o cálculo das fases presentes nas escórias na temperatura de $1400^{\circ} \mathrm{C}$.

As fases formadas nas escórias finais constam na Tabela 7. Vale ressaltar que as simulações foram feitas no FactSage $7.1 \mathrm{com}$ o sub-banco $\mathrm{SlagH}$, sendo o mesmo não apresenta o CaS na forma líquida.

Tabela 7. Composição das fases formadas nas escórias finais dos experimentos de ferro-gusa.

\begin{tabular}{|c|c|c|c|c|c|c|c|}
\hline \multirow{2}{*}{ Misturas } & \multirow{2}{*}{$\begin{array}{c}\text { Sólidos } \\
(\%)\end{array}$} & \multicolumn{4}{|c|}{$\%$ das Fases Sólidas } & \multirow{2}{*}{ Líquidos (\%) } & \multirow{2}{*}{$\eta(\%)$} \\
\hline & & $\mathrm{CaO}$ & $\mathrm{MgO}$ & $\mathrm{Ca}_{3} \mathrm{SiO}_{5}$ & CaS & & \\
\hline Alum $+5 \% \mathrm{CaF}_{2}$ & 87,66 & 86,9 & 0 & 0 & 0,78 & 12,34 & 96,17 \\
\hline $5 \% \mathrm{CaF}_{2}$ & 87,72 & 87 & 0 & 0 & 0,72 & 12,28 & 95,33 \\
\hline 5\% Barrilha & 95,52 & 91,2 & 0,48 & 3,16 & 0,64 & 4,48 & 68,85 \\
\hline $5 \%$ Sodalita & 89,17 & 82 & 0 & 6,83 & 0,37 & 10,83 & 45,83 \\
\hline
\end{tabular}

Pela Tabela 7, pode se notar que nos fundentes que tiveram o pior resultado (barrilha e sodalita), houve a formação de $3 \mathrm{CaO} \mathrm{SiO}_{2}$. Desta forma, é possível inferir que as 
melhores misturas, serão aquelas que, na temperatura de trabalho, tiverem menor porcentagem das fases $\left(3 \mathrm{CaO} . \mathrm{SiO}_{2}\right)$ e $\left(3 \mathrm{CaO} \cdot \mathrm{Al}_{2} \mathrm{O}_{3}\right)$.

Já a mistura contendo barrilha não apresentaram o resultado esperado, uma vez que o $\mathrm{Na}_{2} \mathrm{O}$, produto da decomposição do carbonato de sódio, gera uma escória de maior basicidade e consequentemente, com maior capacidade de sulfeto e partição de enxofre que as escórias de fluorita. Além disso, a decomposição do carbonato de sódio pode elevar a agitação do banho metálico devido a liberação de $\mathrm{CO}_{2}$.

Uma das possíveis causas para a menor eficiência da barrilha pode ser relacionada a reação de parte $\mathrm{Na}_{2} \mathrm{O}$ utilizado com o carbono do cadinho de grafite utilizado nos testes, uma vez que na temperatura dos testes deste trabalho é termodinamicamente possível. Alguns autores, como Iwai e Kunisad [9], Pak e Fruehan [10], afirmam que é possível o carbono reduzir tanto o $\mathrm{Na}_{2} \mathrm{CO}_{3}$ quanto o $\mathrm{Na}_{2} \mathrm{O}$ gerando sódio gasoso.

\subsection{Cinética da dessulfuração}

De posse dos resultados dos testes experimentais, pode-se traçar as curvas $-\ln \left(\frac{\left[\% S_{0}\right]-\left[\% S_{e q}\right]}{\left[\% S_{t}\right]-\left[\% S_{e q}\right]}\right) \times x$ e verificar suas correlações, de modo a identificar que, se para as escórias que formam fases sólidas, existe uma limitação cinética devido ao crescimento desses compostos sólidos sob a partícula de $\mathrm{CaO}$. Os resultados podem ser vistos na Figura 6.

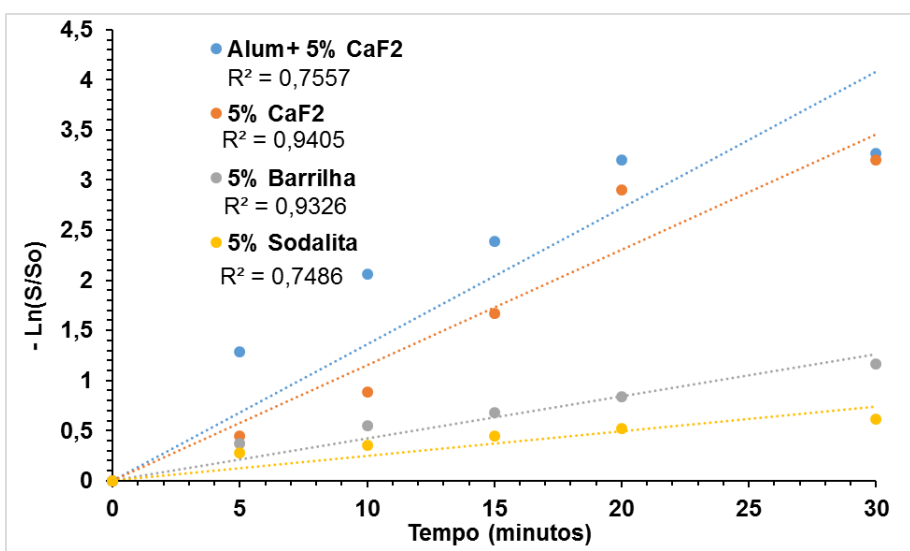

Figura 6. Correlação entre a constante cinética proposta por Frueham e os resultados experimentais.

Pela Figura 6 observa-se que ouve uma baixa correlação da constante de velocidade para os testes experimentais. Na mistura com 5\% de Sodalita, que houve a maior formação de fase sólida, obteve-se a pior correlação. Isso ocorreu, provavelmente, pois segundo Lindstron et al [5], a constante de velocidade passa a ser função da espessura da camada de sólidos formada envolta da partícula de $\mathrm{CaO}$, alterando as propriedades cinéticas e limitam a reação.

Entretanto, as simulações apontaram que na mistura de gusa com Alumínio + 5\% de fluorita, não há formação de $3 \mathrm{CaO}^{-\mathrm{SiO}_{2}}$, apenas fase líquida. Sendo assim, o esperado seria que a equação proposta por Fruehan apresentasse uma melhor correlação, mas não é o que acontece. Sendo assim, não pode ser identificado qual equação para o cálculo da constante cinética pudesse ser usada para este trabalho, sendo que isso é um indício que é necessário um estudo mais aprofundado para determinar uma equação de $k$ que se adeque para a formação de fases sólidas as quais podem ter crescimento com o tempo. 


\section{CONCLUSÃO}

Com base nas condições e nos resultados obtidos no presente estudo, pode-se concluir que:

- As misturas com 5\% de fluorita, apresentaram uma maior eficiência de dessulfuração, com $96,17 \%$ para a mistura com $0,1 \%$ de alumínio no gusa e $95,93 \%$ na escória com apenas cal e $5 \%$ de fluorita;

- A utilização de $5 \%$ de sodalita como fundente não gerou uma boa dessulfuração, uma vez que a grande quantidade de sílica presente provocou a formação de $3 \mathrm{CaO} . \mathrm{SiO}_{2}$, que prejudica a remoção de enxofre;

- A reação de dessulfuração só é endotérmica para valores abaixo de 0,022\% de silício, sendo exotérmica para teores acima desse valor;

- O aumento de temperatura melhora a cinética do processo de dessulfuração de gusa pois promove a redução dos compostos sólidos que prejudicam o processo;

- Não houve uma boa correlação entre os dados experimentais e a constante de velocidade proposta por Fruehan, sendo que há indícios de que se deva levar em conta o crescimento da camada de sólido em volta da partícula de cal. Sendo assim, há uma necessidade de se estudar melhor esse caso.

\section{Agradecimentos}

Agradecimento ao apoio fornecido pela CAPES, FAPES, CNPq e ao IFES.

\section{REFERÊNCIAS}

1 ANDERSON, M. A. T.; JONSSON, P.G.; HALLBERG, M. Optimization of ladle slag composition by application of sulphide capacity model. Ironmaking and Steelmaking, n. 4, v. 27, p. 286-293, 2000.

2 McFEATERS, L.B.; FRUEHAN, R.J. Desulfurization of Bath Smelter Metal. Metallurgical and Materials Transactions B. v. 24B, p. 441-447, 1993.

3 NIEDRINGHAUS, J. C.; FRUEHAN, R. J. Reaction Mechanism for the CaO-Al and CaOCaF2 Desulfurization of Carbon-Saturated Iron. Metallurgical Transactions B, v. 19B, p. 261-268, 1988.

$4 \mathrm{CHOI}, \mathrm{J} . ; \mathrm{KIM}, \mathrm{D}$.; LEE, H. Reaction Kinetics of Desulfurization of Molten Pig Iron Using CaO-SiO2-Al2O3-Na2O Slag Systems. ISIJ International, n. 3, v. 41, p. 216-224, 2001. LINDSTRÖM, D.; NORTIER, P.; Du SICHEN. Kinetic Study on Desulfurization of Hot Metal Using $\mathrm{CaO}$ and $\mathrm{CaC2}$. Metallurgical and Materials Transactions B, v. 46, n. 1, p. 83-92, 2015.

6 LINDSTRÖM, D.; NORTIER, P.; Du SICHEN. Functions of $\mathrm{Mg}$ and $\mathrm{Mg}-\mathrm{CaO}$ Mixtures in Hot Metal Desulfurization. Steel Research int. v. 85, n. 1, p. 76-88, 2014.

7 GRILLO, F. F. Estudo da substituição da fluorita por alumina ou sodalita e de cal por resíduo de mármore em escórias sintéticas dessulfurantes. Dissertação de Doutorado (Departamento de Engenharia Metalúrgica e de Materiais), Escola Politécnica da Universidade de São Paulo (USP), 2015, pp. 100-120.

8 OLIVEIRA, J. R.; SAMPAIO, R. A.; BROSEGHINI, F. C.; OLIVEIRA, H. C. C.; GRILLO, F. F.; VIANA, E. A. Use of Computational Thermodynamic Software in Study of Desulfurizing Mixtures of Hot Metal. AISTech 2014 Proceedings, p. 1207-1212, 2014.

9 IWA, H.; KUNISAD, K. Desulfurization and Simultaneous Dephosphorization of Molten Iron by Na2O-SiO2 and Na2O-CaO-SiO2 Fluxes. ISIJ Interndtional, v. 29, n. 2, p. 135139, 1989.

10 J. J. PakR. J. Fruehan. Dynamics of the hot metal dephosphorization with Na2O slags. Metallurgical Transactions B. 1987, V. 18, 4, 687-693 